\title{
Crossover between Mean-Field and Ising Critical Behavior in a Lattice-Gas Reaction-Diffusion Model
}

\author{
Da-Jiang Liu \\ Ames Laboratory (USDOE), Iowa State University, \\ Ames, Iowa 50011; e-mail: dajiang@fi.ameslab.gov \\ N. Pavlenko \\ Institut für Physikalische Chemie und Elektrochemie, \\ Universität Hannover, Callinstr. 3-3a, D-3016\%, Hannover, Germany \\ J. W. Evans \\ Ames Laboratory (USDOE) and Department of Mathematics, Iowa State University, Ames, Iowa 50011
}

(Dated: October 29, 2018)

\begin{abstract}
Lattice-gas models for $\mathrm{CO}$ oxidation can exhibit a discontinuous nonequilibrium transition between reactive and inactive states, which disappears above a critical CO-desorption rate. Using finite-size-scaling analysis, we demonstrate a crossover from Ising to mean-field behavior at the critical point, with increasing surface mobility of adsorbed $\mathrm{CO}$ or with decreasing system size. This behavior is elucidated by analogy with that of equilibrium Ising-type systems with long-range interactions.
\end{abstract}

\section{INTRODUCTION}

Nonequilibrium phase transitions and pattern formation occur in a broad variety of physical, chemical, biological, and sociological systems $\frac{1,2}{2}$ Such complex behavior sometimes resembles more familiar phenomena in equilibrium systems. However, there are also some fundamental differences due in part to the lack of free energy minimization or detailed-balance constraints for nonequilibrium systems. The latter feature also provides a challenge for development of a rigorous theoretical framework. Nonetheless, the spatial-temporal behavior of nonequilibrium transitions can be effectively studied using one of two paradigms. The first is through a set of partial differential equations (e.g., reaction-diffusion equation for chemical reactions), where phase transitions are related to bifurcations of the underlying nonlinear dynamics.$\frac{1.2}{b}$ By default, criticality is governed by mean-field (MF) behavior, although Ginzburg criteria can be developed to characterize noise-dominated regimes $\stackrel{3}{\underline{3}}$ The second approach utilizes microscopic interacting particle systems of which nonequilibrium lattice-gas models form an important subclass. For these models, one can successfully apply techniques such as finite-size-scaling, and concepts including that of universality classes familiar from critical phenomena in equilibrium phase transitions ${ }^{4}$

Many types of nonequilibrium phase transitions have been studied: continuous transitions to absorbing states 4 (which are often in the universality class of directed percolation), discontinuous transitions between reactive and inactive states,$\frac{5.6}{,}$ and order-disorder transitions, $\frac{7}{7}$ all of which occur in adsorption-desorption or reaction models; driven lattice-gases.8 (where the driving breaks detailed balance); dynamic Ising models with both spin flip and spin exchange dynamics, but at two different temperatures leading to interesting crossover phenomena $\stackrel{\underline{9}}{\underline{p}}$ For some of these systems, it is possible to derive rigorous reaction-diffusion equations in the hydrodynamic limit of rapid exchange or diffusion $\underline{6.10}$

In this paper, we consider specifically surface reaction models, which incorporate adsorption-desorption and reaction steps, as well as surface mobility. Our motivation is that these models not only contain the essential ingredients to elucidate complex phenomena observed in real surface catalysis, but also exhibit many fascinating features of the above idealized models. On the one hand, they exhibit complex dynamics and phase transitions associated with the reaction mechanism (and perhaps with adspecies interactions), but also surface diffusion rates provide a key parameter which can tune behavior. In fact, while many theoretical studies have considered the regime of limited or zero mobility, it is the hydrodynamic regime of rapid mobility that is most often realized in experiments $\underline{\underline{6}}$

Our focus here is on first-order phase transitions and associated critical phenomena in models for $\mathrm{CO}$ oxidation on metal surfaces, behavior analogous to that of the ferromagnetic Ising model. The central question is "how does critical behavior depend on surface mobility", anticipating that MF behavior could well apply in the hydrodynamic regime, but that a crossover may occur to non-MF behavior for lower mobility. Through precise finite-size-scaling (FSS) studies of simulation data, we confirm this prediction and quantify crossover behavior. We also comment on experimental realization of crossover for $\mathrm{CO}$ oxidation in high-pressure nanoscale systems.

\section{REACTION MODEL: SPECIFICATION AND BEHAVIOR}

We now describe our lattice-gas reaction-diffusion model, where gas (ads) denote gas-phase (adsorbed- 
phase) species. The following steps are implemented using a square lattice of adsorption sites with periodic boundary conditions. (i) $\mathrm{CO}$ (gas) adsorbs on single empty sites at rate $p=p_{\mathrm{CO}}$ (chosen between 0 and 1) and desorbs with rate $d$. (ii) $\mathrm{O}_{2}$ (gas) adsorbs dissociatively onto a pair of diagonally adjacent empty sites at rate $p_{\mathrm{O}_{2}}=1-p_{\mathrm{CO}}$, provided all six neighbors are free of $\mathrm{O}$ (ads). This "eight-site" rule reflects strong nearest-neighbor $(\mathrm{NN}) \mathrm{O}(\mathrm{ads})-\mathrm{O}(\mathrm{ads})$ repulsions. Since $\mathrm{O}$ (ads) is treated as immobile, this adsorption rule ensures that $\mathrm{O}$ (ads) never occupy adjacent sites. (iii) adjacent $\mathrm{CO}$ (ads) and $\mathrm{O}$ (ads) react at rate $k$ (set to unity here). (iv) $\mathrm{CO}$ (ads) hops to adjacent empty sites at rate $h$. This model has also been discussed elsewhere, e.g., Refs. 67.

Conventional kinetic Monte Carlo (KMC) simulations are used to assess model behavior for finite $h$. Noting that mobility of $\mathrm{CO}$ (ads) is often very high under ultrahigh vacuum conditions, we also perform a direct analysis of limiting behavior for $h=\infty$ using a "hybrid" treatment: here the distribution of $\mathrm{O}(\mathrm{ads})$ is described within a lattice-gas framework, but one only tracks the number of $\mathrm{CO}$ (ads) and assumes that they are randomly distributed on sites not occupied by $\mathrm{O}(\mathrm{ads}) \underline{\underline{\underline{6}}}$ For very large $h$ and low O-coverages, this is valid.

We now briefly review the steady state behavior of this model for an infinite system. First, consider the case of finite $h<\infty$, ${ }^{5.6}$ For low $d$, one typically finds a first-order transition at some $p=p_{\mathrm{CO}}=p^{*}$ between a reactive state with low CO-coverage $\left\langle\theta_{\mathrm{CO}}\right\rangle$ (for $p<p^{*}$ ) and an inactive state with high $\left\langle\theta_{\mathrm{CO}}\right\rangle$ (for $\left.p>p^{*}\right)$. The transition disappears as $d$ increases above some critical value $d_{c}(h)$. Second, consider the hybrid model with $h=\infty \frac{6.7}{}$ Here one finds a region of bistability with both reactive and inactive states (the discontinuous transition at $p=p^{*}$ for finite $h$ corresponding to the equistability point). Bistability disappears at a cusp bifurcation upon increasing $d$ above some $d_{c}(\infty)$. A coherent picture for both cases of infinite and finite $h$ comes from the observation that decreasing $h$ decreases the degree of metastability or hysteresis in the system.

Next, we describe how behavior changes for finite $L$. For the reaction model with finite $h$, the discontinuous transition in the $\left\langle\theta_{\mathrm{CO}}\right\rangle$ versus $p_{\mathrm{CO}}$ mentioned above is rounded for finite $L$, but becomes sharper as $L$ increases. The trend is analogous to behavior for the equilibrium Ising model in finite systems. In the hybrid model with $h=\infty$, there is also a smooth transition in the $\left\langle\theta_{\mathrm{CO}}\right\rangle$ versus $p_{\mathrm{CO}}$ for finite $L$. This reflects the feature that for $L<\infty$, the system can make noise-induced jumps between the low $\left\langle\theta_{\mathrm{CO}}\right\rangle$ reactive and high $\left\langle\theta_{\mathrm{CO}}\right\rangle$ inactive states, and that the relative weight of these states changes smoothly with $p_{\mathrm{CO}}$. This transition also becomes sharper with increasing $L$, occurring at the equistability point for $L \rightarrow \infty$. See Ref. 11 for details.

Finally, to place our study in a broader context, we note that our CO oxidation model corresponds to a modified version of the Ziff-Gulari-Barshad (ZGB) model, ${ }^{5}$ and its extension with desorption of $\mathrm{CO}$ (ads). The key modifications in our model are inclusion of: a) hopping of $\mathrm{CO}(\mathrm{ads})$; b) $\mathrm{NN}$ exclusion of $\mathrm{O}(\mathrm{ads})$; and c) finite rather than infinite reaction rate. All these features are important for realistic modeling of $\mathrm{CO}$ oxidation. However, it is primarily the first feature [hopping of $\mathrm{CO}(\mathrm{ads})]$ which impacts the critical behavior of the reactive-inactive transition studied in this paper. Furthermore, it is clear that our conclusions about critical behavior would apply to the ZGB model, modified to include desorption and hopping of $\mathrm{CO}$ (ads). The second feature [NN exclusion of $\mathrm{O}(\mathrm{ads})]$ results in an oxygen poisoning transition in the ZBG mode ${ }^{5}$ being replaced by an order-disorder transition ${ }^{7}$ This does not affect critical behavior of the reactive-inactive transition, with one caveat. For small $h$, NN exclusion does also lead to a loss of the reactiveinactive transition (see Sec. IV). The third modification to the original ZGB model is that instead of $k=\infty$, we choose $k=1$, i.e., the reaction rate equals the total adsorption rate. The choice is of course somewhat arbitrary, but the basic behavior of these models does not change varying the reaction rate from $O(1)$ to $\infty$. For further discussion of effects of varying $k$ on the steady state bifurcation diagram, see Ref. 12 .

\section{CRITICAL POINT DETERMINATION AND FSS ANALYSIS}

Unlike the Ising model, transitions in reaction model do not involve simple symmetry-breaking. As in the liquid-vapor phase separation problem, one needs to locate the critical point in a two parameter space, i.e., $(p, d)$ for the reaction model. However here one also has the disadvantage that computationally efficient techniques for equilibrium systems (e.g., histogram-reweighting and cluster algorithms) do not apply. Thus, when using numerical techniques such as FSS, careful analysis of simulation data is necessary. Below, we briefly describe our procedure.

There are several reasonable ways to define the effective transition point $p=p_{L}^{*}$ for finite $L$. One natural choice is the point where the change in the relevant order parameter (i.e., the $\mathrm{CO}$ coverage $\left.\left\langle\theta_{\mathrm{CO}}\right\rangle\right)$ is greatest. All choices should converge to the same value as $L$ diverges (for fixed $d<d_{c}$ ), and should yield the same critical exponents in the FSS analysis described below. For a given $d<d_{c}$, we measure CO-coverage $\left\langle\theta_{\mathrm{CO}}\right\rangle$ versus $p$ for two different system sizes, say $L$ and $2 L$. We find that a convenient definition of $p_{L}^{*}$ is the point where the curves cross, i.e., where $\left\langle\theta_{\mathrm{CO}}\left(p_{L}^{*}\right)\right\rangle_{L}=\left\langle\theta_{\mathrm{CO}}\left(p_{L}^{*}\right)\right\rangle_{2 L}$. The technique is similar in its underlying motivation to a method for equilibrium first-order transitions by Borgs and Janke 13

After determining the transition pressure $p^{*}$ for each $d$ value, one can study the critical behavior using the usual FSS techniques. For example, the quantity $\chi_{L} \equiv$ $L^{2}\left(\left\langle\theta_{\mathrm{CO}}^{2}\right\rangle-\left\langle\theta_{\mathrm{CO}}\right\rangle^{2}\right)$, which is related to susceptibility in 
equilibrium systems, is assumed to have the following behavior

$$
\chi_{L}=L^{\gamma / \nu} \tilde{\chi}_{\operatorname{Ising}}\left[\left(d-d_{c}\right) L^{1 / \nu}\right],
$$

if the transition belongs to the Ising universality class. Here $\nu=1$ and $\gamma=7 / 4$ are the critical exponents for the correlation length and susceptibility respectively (in 2D). In contrast, for the MF universality class, one has ${ }^{14.15}$

$$
\chi_{L}=L^{D / 2} \tilde{\chi}_{\mathrm{MF}}\left[\left(d-d_{c}\right) L^{D / 2}\right],
$$

where $D=2$ is the spatial dimension of the system so that $L^{D}$ is the volume (or area in our case) of the system. Defining $R_{L} \equiv \chi_{2 L} / \chi_{L}$, in either case, one has

$$
R_{L}\left(d_{c}\right) \rightarrow 2^{\tilde{\gamma}}, \text { as } L \rightarrow \infty
$$

where the size scaling exponent $\tilde{\gamma}$ is $D / 2=1$ for the MF universality class, and $\gamma / \nu=7 / 4$ for the Ising universality class.

Since one does not know the value of $d_{c}$ a priori, a convenient way to determine both the critical point and exponent is by finding the crossing point of $R_{L}(d)$ and $R_{2 L}(d)$, so that

$$
R_{L}\left(d_{c}^{L}\right)=R_{2 L}\left(d_{c}^{L}\right)=2^{\tilde{\gamma}_{L}} .
$$

$\tilde{\gamma}_{L}$ can be considered as the effective critical exponent for finite systems (see Sec. [V]). Note that in determining $d_{c}^{L}$ and $\tilde{\gamma}_{L}$, we need simulations of system of linear sizes $L$, $2 L$, and $4 L$.

Figure 1shows $R_{L}$ versus $d$ for the hybrid model. The FSS argument above predicts that $R_{L}$ for different $L$ 's will cross at $\left(d_{c}, R_{c}\right)$ where $R_{c}=2$ for MF criticality, and $R_{c}=2^{7 / 4} \approx 3.364$ for Ising (in $2 \mathrm{D}$ ) criticality. Figure 1 clearly shows MF behavior for system up to $L=128$. Note that in the limit of $L \rightarrow \infty, R_{L}$ is a step function with $R_{L}=4$ when $d<d_{c}$, and $R_{L}=1$ when $d>d_{c}$

Table I lists the effective critical exponent obtained using the above procedure for the hybrid model. All estimates for $\tilde{\gamma}_{L}$ are close to unity, consistent with the prediction of MF universality class where $\tilde{\gamma}=1, \underline{14}$ Extrapolation to $L=\infty$ assuming a $1 / L^{2}$ finite size correction (consistent with MF criticality) gives $d_{c}=0.05258(5)$ and $p_{c}=0.41327(5)$. Assuming a $1 / L$ finite size correction for $\tilde{\gamma}_{L}$ gives $\tilde{\gamma}=0.995(6)$. It is possible that corrections to scaling are described by other exponents. However, our analysis shows that the range of system sizes is large enough so that the value of $d_{c}$ and the consistency with MF behavior is not dependent on the form of the corrections. Note that MF behavior occurs despite the presence of spatial correlations in the distribution of $\mathrm{O}(\mathrm{ads})$ due to limited mobility of $\mathrm{O}(\mathrm{ads})$ and interactions between $\mathrm{O}(\mathrm{ads}) \stackrel{?}{\underline{\underline{T}}}$

Figure 2] shows $R_{L}$ versus $d$ for the reaction model with finite $h=1$. The crossing point appears to approach $2^{7 / 4}$ as $L$ increases, consistent with the prediction of Ising universality. Limitations in analysis of larger system sizes

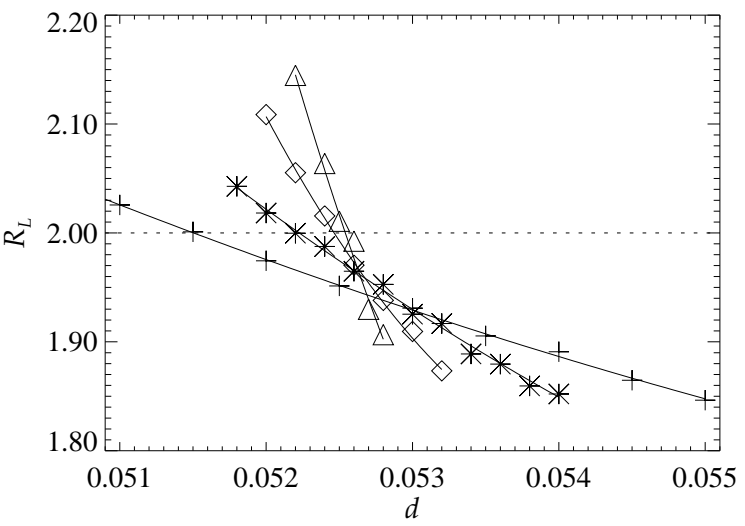

FIG. 1: Ratio, $R_{L}$, of mean-square fluctuation amplitudes for systems of size $2 L$ and $L$ at equistability points for the hybrid model. $L$ ranges from $8,16,32$ to 64 with increasing steepness. The dotted line plot $R_{L}=2$, the MF prediction.

TABLE I: Effective critical point and critical exponent for fluctuations of $\theta_{\mathrm{CO}}$ for the hybrid model obtained from finitesize-scaling analysis.

\begin{tabular}{cccc}
\hline \hline$L$ & $d_{c}$ & $p_{c}$ & $\tilde{\gamma}_{L}$ \\
\hline 8 & $0.05304(6)$ & $0.4138(1)$ & $0.948(2)$ \\
16 & $0.05275(5)$ & $0.41343(8)$ & $0.968(4)$ \\
32 & $0.05264(3)$ & $0.41330(6)$ & $0.981(5)$ \\
\hline \hline
\end{tabular}

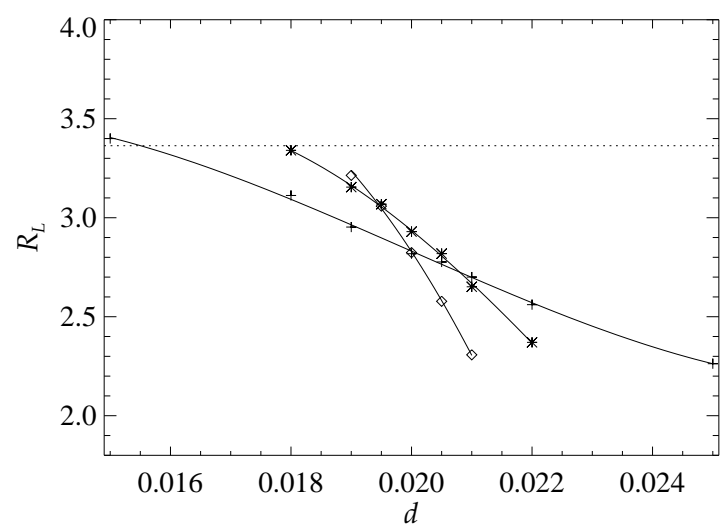

FIG. 2: Ratio, $R_{L}$, of mean-square fluctuations amplitudes for systems of size $2 L$ and $L$ at equistability points for the reaction model with finite $\mathrm{CO}$ diffusion model $h=1$. $L$ ranges from 8,16 , and 32 with increasing steepness. The dotted line shows $R_{L}=2^{7 / 4}$, the Ising prediction.

precludes definitive convergence of FSS results for either the hybrid model (Fig. 10 or finite $h$ (Fig. 2 However, comparing these cases reveals very different behavior, and we argue that the above assignment of universality classes is quite reasonable and natural. 
TABLE II: Variation of the critical desorption rate $d_{c}(h)$ with CO hop rate $h$. Results are obtained from FSS scaling using system sizes $L=16,32$, and 64 .

\begin{tabular}{cccccccc}
\hline \hline$h$ & 0.5 & 1 & 2 & 4 & 10 & 20 & $\infty$ \\
\hline$d_{c}(h)$ & 0.012 & 0.019 & 0.027 & 0.035 & 0.042 & 0.047 & 0.0527 \\
\hline \hline
\end{tabular}

From the slope of $R_{L}$ at the crossing point and the scaling form $\tilde{\chi}\left[\left(d-d_{c}\right) L^{\theta}\right]$ in Eqs. (1) and (2) one can also estimate the exponent related to the rounding of the critical region due to finite sizes. Using results in Fig. [1 we find that $\theta=1.0(1)$ for the largest system sizes, consistent with the MF value $\theta=D / 2=1$. Uncertainties in the data of Fig. 2 are too large to obtain accurate estimate of $\theta$, but they are consistent with the Ising value $1 / \nu=1$. In two dimensions, $\theta$ can not be used to distinguish between the MF and Ising universality class.

We note one previous study by Tomé and Dickman 17 of the ZGB lattice-gas mode $1^{5}$ for $\mathrm{CO}$ oxidation $\left(\mathrm{O}_{2}\right.$ adsorption on adjacent sites, infinitely fast reaction, no $\mathrm{CO}$ diffusion) modified to include CO-desorption. They measure the shift of the critical point with system size and found that $d_{c}(L)-d_{c}(\infty) \sim L^{-\lambda}$ where $\lambda=1$ consistent with results of the two-dimensional Ising model. Indeed, using a similar method, we found that $\lambda \approx 1$ for $h=1$ and $\lambda \approx 2$ for the hybrid model $\frac{18}{n}$ This result is consistent with our conclusion regarding universality classes. However, care must be taken with the interpretation of the shift exponent, $\lambda$, because it is sensitive to the boundary conditions (which were chosen to be periodic in both the above studies) in the case of MF universality where hyperscaling is violated $\stackrel{16}{ }$ For example, choosing free boundary conditions (which modifies adsorption and reaction processes near the boundaries) could lead to modified $\lambda$ for the hybrid model.

\section{CROSSOVER BEHAVIOR}

Table凹shows the variation with $h$ of the critical point $d_{c}$ for the CO poisoning transition obtained using FSS. The $h=\infty$ value is taken from the hybrid model. By analogy with equilibrium studies, discussed below and in Ref. 14, we assume that the shift of $d_{c}(h)$ away from the limit of $h \rightarrow \infty$ scales as $1 / h$ (although there could be logarithmic corrections).

As an interesting aside, we note that extrapolation behavior to the regime of small $h$ suggests that $d_{c}(h)$ vanishes at $h=h_{t} \approx 0.2$. Thus, for small $0<h<h_{t}$, the $\mathrm{CO}$ poisoning transition is continuous for $d=0$, and does not exist for $d>0$. This is in contrast to the ZGB model where a first-order $\mathrm{CO}$ poisoning transition exists even for immobile CO. For the case of $d=0$ and $h<h_{t}$, our epidemic analysis (cf. Ref. [4) indicates that the transition to the absorbing ( $\mathrm{CO}$ poisoned) state, belongs to the directed percolation universality class.
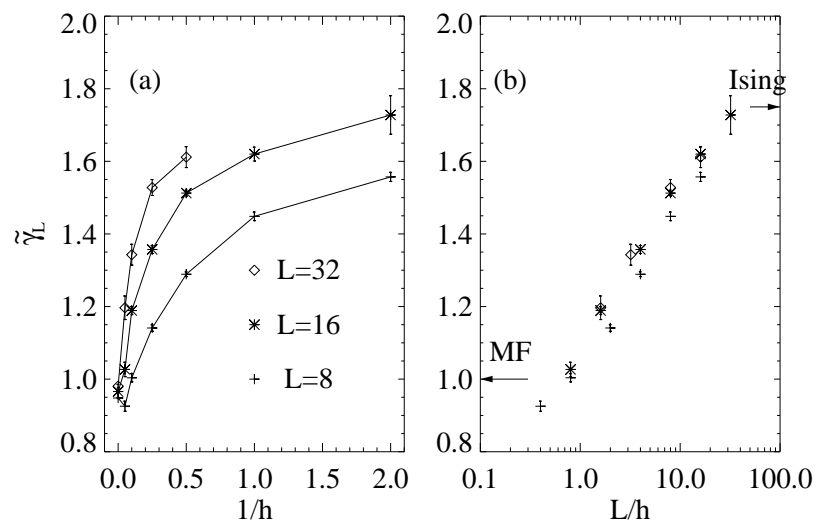

FIG. 3: Effective critical exponent $\tilde{\gamma}$ versus $h$. See text for details.

Thus, to summarize our results for the critical point in our reaction model with $k=1$, we find that the critical desorption rate $d_{c}$ increases from $d_{c}=0$ for (small) $h=h_{t}$ to $d_{c}=0.0527$ for $h=\infty$. In contrast, for the ZGB model (no NN exclusion of O) with $k=\infty$, modified to include $\mathrm{CO}$ desorption and $\mathrm{CO}$ mobility, one finds that $d_{c}=0.04^{17.19}$ for $h=0$ increasing to $d_{c}=2 / 3$ for $h=\infty .12$ Further refining this ZGB-type model to incorporate $k=1$ (rather than $k=\infty$ ), one finds that $d_{c}=0.03^{20}$ for $h=0$ increasing to $d_{c}=0.142$ for $h=\infty .21$.

Of more central interest to this paper is the variation with $h$ of the (effective) critical exponents. Upon increasing the $\mathrm{CO}$ diffusion rate, $h$, how does one crossover from Ising criticality (applying for finite $h>h_{t}$ ) to the MF criticality of the hybrid model (which corresponds to first taking the limit of $h \rightarrow \infty$ ). A related question is: for finite (possibly large) $h$, how does critical behavior depend on finite system size?

In the literature on crossover studies, effective exponents are usually defined as the slope of the log-log plot of the measured critical quantities versus deviation from the critical point, e.g., $\gamma_{\text {eff }} \equiv-d \ln \chi / d \ln \left|d-d_{c}\right|$. A different definition, which is more amenable to numerical simulations is to fix the parameters at the critical point, while changing the system size $L$. The effective (reduced) exponent can then be defined as $\tilde{\gamma}_{\text {eff }} \equiv d \ln \chi / d \ln L$. In principle, this derivative (or its finite difference approximation) should be evaluated at the critical point for the infinite system, $d_{c}$. In practice, $d_{c}$ is unknown a priori, and estimation by extrapolation produces additional errors (particularly using simulation data for limited system size). Thus instead, one just uses $d_{c}^{L}$ obtained from the FSS analysis in Eq. (4), and therefore $\tilde{\gamma}_{L}$ also from Eq. (4) as the effective exponent.

Figure 3(a) shows $\tilde{\gamma}_{L}$ versus variable $1 / h$ for $h=0.5$, $1,2,4$, and 10 . The system size is indicated in the figure. As Fig. 3(a) shows, $\tilde{\gamma}$ approaches 1 as $h \rightarrow \infty$, and approaches $7 / 4$ when $h$ is small. This behavior can be un- 
derstood in terms of the theoretical framework presented below.

Phenomenological analysis of nonequilibrium dynamics (and more specifically of critical phenomena) in Hamiltonian systems with nonconserved order parameters has traditionally been formulated in terms of timedependent Ginzburg-Landau equations (TDGLE) $\stackrel{22}{=}$ For multi-component order parameter, $\phi_{\alpha}$, the TDGLE has the general form

$$
\frac{\partial \phi_{\alpha}(\mathbf{x}, t)}{\partial t}=-\Gamma \frac{\delta F}{\delta \phi_{\alpha}(\mathbf{x}, t)}+\zeta_{\alpha}(\mathbf{x}, t),
$$

where $\zeta_{\alpha}$ is Gaussian white noise terms. Here $F$ is the coarse-grained free energy functional, which for an Ising-type model (with single-component order parameter) with interactions of range $R$ can be written as ${ }^{14.15}$

$$
F=\int d \mathbf{x}\left[V(\phi)+\frac{1}{2}|R \nabla \phi|^{2}\right] .
$$

The quartic potential $V(\phi)$ converts from double- to single-well form with increasing temperature through the critical point. This type of formulation extends to nonequilibrium reaction systems characterized by a vector $\mathbf{c}$ of adspecies concentrations where the TDGLE are replaced by reaction-diffusion equations (RDE) of the form

$$
\partial \mathbf{c}(\mathbf{x}, t) / \partial t \propto-\partial V_{\mathrm{eff}}(\mathbf{c}) / \partial \mathbf{c}+\nabla \cdot \mathrm{D} \cdot \nabla \mathbf{c}+\zeta .
$$

Analogous to $V$, the effective potential $V_{\text {eff }}$ converts from double- to single-well passing through the critical point via the bistable region. In our problem, the diffusion tensor satisfies $\mathrm{D} \propto h$ (the microscopic hop rate) except for small $h \underline{23}^{23}$ Thus, comparing the RDE with TDGLE after performing the functional differentiation, it is clear that $h^{1 / 2}$ plays the role of $R$. This result is reasonable if one recognizes these types of reaction-diffusion models (with finite reaction rate and typically large diffusion rates) exhibit a characteristic diffusion length, $L_{\text {diff }}$, which scales like $L_{\text {diff }} \sim(h / K)^{1 / 2}$. Here, $h$ is the microscopic hop rate, and $K$ is some effective rate for the overall adsorption-reaction process. See Refs. 6 24. Thus, refining the above statement, we can say that $L_{\text {diff }}$ plays the role of $R$. Finally, we also note that from analysis of either the TDGLE or the RDE for infinite systems, it is long known that fluctuations dominate close enough to the critical point for spatial dimension $D<4$.

Recently, extensive studies have been performed on finite size effects in equilibrium Ising-type systems with long-range interactions. From scaling and renormalization procedures,$\frac{14.15}{4}$ as well as numerical simulations, ${ }^{25}$ it has been shown that the crossover from MF to Ising behavior in finite $L \times L$ systems is governed by the ratio $L / R^{2}$. Thus, by analogy, one expects that the crossover for reaction-diffusion systems is governed by $L / h$ (in $2 \mathrm{D}$ ). However, equilibrium and reactions-diffusion systems are quite different in detail: the Hamiltonian formulation, which is central to analysis of the former, does not exist for the latter; the microscopic nature of diffusion is distinct from that of long-range interactions. Thus, it is appropriate to test numerically the above scaling hypothesis. In Fig. 3(b), we replot the data in Fig. 3(a) using scaling variable $L / h$. Data collapse, while imperfect for $L=8$, is quite good for $L>16$, supporting our proposal that that $L / h$ is the correct size scaling variable.

Of course, it would be preferable to set the phenomenological analysis on a more rigorous footing. In general, the strategy of extending mean-field-type formulations to include noise via stochastic partial differential equations can be unreliable. An alternative rigorous approach is to attempt to map the exact master equations for the model onto a stochastic field theory ${ }^{26}$ This strategy has been applied for a variety of toy reaction-diffusion models, e.g., demonstrating that the introduction of diffusion can modify the number of adsorbing states and the universality class in pair-contact models with diffusion ${ }^{27}$ However, these models are quite different from our reaction-limited monomer-dimer type models, where the utility of the rigorous approach has yet to be demonstrated.

\section{CONCLUSIONS}

Catalytic CO oxidation on noble metal surfaces provides a convenient (and important) chemical system for which lattice-gas modeling can be used to rigorously assess criticality in a reaction-diffusion system. We have systematically studied the role that $\mathrm{CO}$ diffusion plays on the criticality of the $\mathrm{CO}$ poisoning transition. We find a crossover from MF criticality for fast diffusion [which occurs despite spatial correlations in the distribution of $\mathrm{O}(\mathrm{ads})]$, to Ising criticality for limited diffusion. The natural variable describing this crossover is proposed based on phenomenological arguments. The proposition is supported by numerical simulations.

Recent experiments on $\mathrm{CO}$ oxidation in nanoscale systems can probe fluctuations and critical behavior ${ }^{28.29}$ Thus, the considerations of this paper, including the study of crossover as a function of system size, become particularly relevant. Furthermore, the crossover behavior described in this study may be realized physically as one makes the transition from low-pressure to highpressure catalysis. The latter could produce a decrease by many orders of magnitude of the diffusion rates (relative to other rates) that results from the higher surface coverages 30

\section{Acknowledgments}

DJL and JWE were supported by the USDOE-BES through Ames Laboratory which is operated for the USDOE by Iowa State University under Contract No. W7405-Eng-82. N.P. gratefully acknowledges the support 
1 H. Haken, Rev. Mod. Phys. 47, 67 (1975).

2 A. S. Mikhailov, Foundations of Synergetics I: Distributed Active Systems (Springer-Verlag, Berlin, 1990).

3 A. Nitzan and P. Ortoleva, Phys. Rev. A 21, 1735 (1980).

4 J. Marro and R. Dickman, Nonequilibrium phase transitions in lattice models (Cambridge University Press, Cambridge, 1999).

5 R. M. Ziff, E. Gulari, and Y. Barshad, Phys. Rev. Lett. 56, 2553 (1986).

6 J. W. Evans, D.-J. Liu, and M. Tammaro, Chaos 12, 131 (2002).

7 D.-J. Liu and J. W. Evans, Phys. Rev. Lett. 84, 955 (2000).

8 B. Schmittmann and R. K. P. Zia, Phys. Rep. 301, 45 (1998).

9 J. M. Gonzalez-Miranda, P. L. Garido, J. Marro, and J. L. Lebowitz, Phys. Rev. Lett. 59, 1934 (1987).

10 A. de Masi, P. A. Ferrari, and J. L. Lebowitz, Phys. Rev. Lett. 55, 1947 (1985).

11 D.-J. Liu and J. W. Evans, J. Chem. Phys. 117, 7319 (2002).

12 J. W. Evans and M. Tammaro, in Computer Simulation Studies in Condensed-Matter Physics, edited by D. P. Landau and H.-B. Schuttler (Springer, Berlin, 1999), vol. XI, p. 103.

13 C. Borgs and W. Janke, Phys. Rev. Lett. 68, 1738 (1992).

14 K. K. Mon and K. Binder, Phys. Rev. E 48, 2498 (1993).

15 E. Luijten, H. W. J. Blöte, and K. Binder, Phys. Rev. E 54, 4626 (1996).

16 V. Privman and M. E. Fisher, J. Stat. Phys. 33, 385 (1983).

17 T. Tomé and R. Dickman, Phys. Rev. E 47, 948 (1993).

18 N. Pavlenko, R. Imbihl, J. W. Evans, and D.-J. Liu, in preparation.

19 B. J. Brosilow and R. M. Ziff, Phys. Rev. A 46, 4534
(1992).

20 J. W. Evans and M. C. Bartelt, Phys. Rev. B 49, 1061 (1994)

21 M. Tammaro, M. Sabella, and J. W. Evans, J. Chem. Phys. 103, 10277 (1995).

22 P. C. Hohenberg and B. I. Halperin, Rev. Mod. Phys. 49, 435 (1977).

${ }^{23}$ For small values of "bare" hop rate $h$, D scales differently $h$, as spatial coupling from two-site adsorption and reaction processes competes with that from diffusion. One expects that $\mathrm{D} \sim c+h$, where $c=O(1)$. See E. Clément, P. Leroux-Hugon, and L. M. Sander, Phys. Rev. Lett. 67, 1661 (1991); J. W. Evans and T. R. Ray, Phys. Rev. E 50, 4302( 1994).

${ }^{24}$ R. Imbihl and G. Ertl, Chem. Rev. 95, 697 (1995).

25 E. Luijten, H. W. J. Böte, and K. Binder, Phys. Rev. E 56, 6540 (1997).

26 U. C. Täuber, Acta Physica Slovaca 52, 505 (2002), condmat/0205327.

27 H. Hinrichsen, Adv. Phys. 49, 815 (2000).

${ }^{28}$ Y. Suchorski, J. Beben, E. W. James, J. W. Evans, and R. Imbihl, Phys. Rev. Lett. 82, 1907 (1999).

29 Y. Suchorski, J. Beben, R. Imbihl, E. W. James, D.-J. Liu, and J. W. Evans, Phys. Rev. B 63, 165417 (2001).

30 In real reaction systems, we expect that the chemical diffusion rates (rather than the microscopic hop rates) controls crossover. Increased coverage does not necessarily reduce chemical diffusivity in single species adlayers. However, it is generally expected to reduce chemical diffusivity in mixed adlayers due to effects of percolation and interactions, see e.g., D.-J. Liu and J. W. Evans, J. Chem. Phys. 113, 10 $252(2000)$. 